\title{
A propósito del covid-19: ¿Sería recomendable para Chile la mediación familiar en línea?
}

\author{
About Covid-19: Would the online family mediation \\ be recommended for Chile?
}

\author{
Sofía Alarcón García \\ Abogada independiente, Chile
}

\begin{abstract}
RESUMEN En este estudio analizamos si la mediación en línea es recomendable para el Sistema de Mediación Familiar de Chile. La hipótesis que planteamos fue que la mediación en línea puede beneficiar a los usuarios y mediadores de dicho sistema, y que no impide desempeñar de manera adecuada la labor mediadora o cumplir los principios del proceso. Los resultados mostraron que dicha modalidad puede favorecer el acceso a la mediación y evitar la discontinuidad del servicio cuando no es posible reunir físicamente a los participantes, y que, además, no impide el desempeño adecuado de la labor mediadora o cumplir los principios del proceso. Por ello, concluimos que sí es recomendable su implementación y que no es razonable que sólo se admita la concurrencia física.
\end{abstract}

PALABRAS CLAVE Mediación familiar chilena, mediación en línea, mediación en Chile, covid-19, Ley 21.226.

ABSTRACT In this study, we analyzed whether online mediation is recommended for the Chilean Family Mediation System. The hypothesis that we proposed was that online mediation could benefit the users and mediators of said System, and would not prevent the mediator from adequately performing the mediation work or complying with the principles of the process. The results showed that this modality could favor access to mediation and avoid the discontinuity of the service when it is not possible to physically gather the participants, and furthermore, it would not hinder the adequate performance of the mediation work nor would it comply with the principles of the process. Therefore, we conclude that it is not reasonable that only physical assistance be admitted.

KEYWORDS Chilean family mediation, online mediation, mediation in Chile, Covid-19, Law 21.226. 


\section{Introducción}

En el mundo se han adoptado medidas extraordinarias para mitigar las tasas de contagio y letalidad de la enfermedad covid-19. ${ }^{.}$Estas medidas han transformado distintos ámbitos de la vida cotidiana, incluyendo el funcionamiento de las instancias de administración de justicia. ${ }^{2}$ El 2 de abril de 2020 se publicó en Chile la Ley 21.226. Esta ley inició un régimen jurídico de excepción por el impacto del covid-19. Una de las materias que modificó fue la obligatoriedad que dispone la Ley 19.968 para los procesos de mediación familiar. Por mandato del artículo 106, inciso primero de la Ley 19.968, los interesados en interponer una demanda por pensión de alimentos, relación directa y regular, o cuidado personal de niños y adolescentes, deben acreditar previamente haber intentado un proceso de mediación. La Ley 21.226 en su artículo 8 , inciso cuarto, eximió la obligación de la mediación previa antes señalada y, en cambio, facultó a los interesados para intentar de manera voluntaria un proceso de mediación previo. No obstante, a causa de la contingencia sanitaria y la declaración de zonas de cuarentena, los centros de mediación familiar de Chile se vieron obligados a cesar paulatinamente su funcionamiento presencial. En este mismo contexto, otros servicios públicos en Chile comenzaron a utilizar tecnologías digitales para mantener la continuidad de los servicios prestados. Pese a esta situación, las autoridades de la Unidad de Mediación y Resolución Alternativa de Conflictos de la División Judicial del Ministerio de Justicia y Derechos Humanos descartaron que fuera posible hacer mediaciones en línea. Las autoridades fundamentaron la prohibición indicando que la mediación en línea era incompatible con la concurrencia personal que exige el artículo 108 de la Ley 19.968. ${ }^{3}$ Además, indicaron que la mediación en línea no era recomendable para el Sistema Nacional de Mediación Familiar por factores geográficos, sociales y económicos, y porque sólo la mediación presencial permite cumplir en forma adecuada la labor mediadora y cumplir los principios de la mediación. Por ello, las autoridades de la Unidad concluyeron que aun existiendo los medios para que los usuarios accedan a la mediación en línea, permitirla significaría atentar en contra de la institución. ${ }^{4}$ No obstante, si por un lado se exige que las mediaciones sean presenciales, pero por el otro, ni los mediadores o los usuarios pueden asistir a

1. Allegra Frank y Constance Grady, «Phone booths, parades, and 10-minute test kits: How countries worldwide are fighting Covid-19», Vox, 22 de marzo de 2020, disponible en https://bit.ly/2BeYruf.

2. Diego García-Sayán, «Y ante el coronavirus, la justicia, ¿qué?», El País, 16 de abril de 2020, disponible en https://bit.ly/3d5Wwp6; Aura Esther Vilalta Nicuesa, «El acceso a la justicia en tiempos del covid-19», The Conversation, 29 de abril de 2020, disponible en https://bit.ly/2Y3rvOn.

3. Unidad de Mediación y Resolución Alternativa de Conflictos, «Pronunciamiento sobre realización del proceso de mediación a distancia», Mediación de Conflictos Familiares, 25 de marzo de 2020, p. 2, disponible en https://bit.ly/2YBcy5p.

4. Unidad de Mediación, «Pronunciamiento...», 4-6. 
los centros de mediación, la prohibición de la autoridad lleva a que las familias chilenas queden privadas de acceder a la justicia familiar colaborativa. Si los usuarios no tienen cómo solucionar los impedimentos de acceso a la mediación familiar, esta situación, además de afectar a las familias, podría llevar a una sobrecarga significativa en el sistema judicial.

Los primeros proyectos de resolución de conflictos en línea se ejecutaron en Estados Unidos durante la década de los noventa (Ortega, 2019: 55). En 2011 ya existía una variada oferta de proveedores de servicios de mediación en línea dentro de América del Norte, Oceanía y Europa (Poblet y otros, 2011: 966-970). En principio, esta modalidad sólo se empleó para tratar disputas que surgían en internet, pero más tarde también comenzó a utilizarse en los conflictos que se originaban fuera de la red o dentro de una misma zona geográfica (Alzate, 2008: 9; Lavi, 2015: 260). Durante la última década, en países como España, Reino Unido, Estados Unidos y Australia, la mediación en línea se utilizó para resolver conflictos de distinta naturaleza, como del ámbito empresarial, comercial, familiar, comunitario y de propiedad intelectual (Poblet y otros, 2011: 968-972, 978-980). Sin embargo, el mayor auge de la mediación en línea se experimentó a causa de la pandemia covid-19. Para dar continuidad a los servicios de mediación y no arriesgar la salud pública, distintos países optaron por suspender las mediaciones presenciales y permitir sólo la mediación en línea. Esto ocurrió incluso en países como México y Argentina, pese a que en ellos todavía era una modalidad incipiente. ${ }^{5}$

Como consecuencia, nos preguntamos si es correcto afirmar de manera categórica que la mediación en línea no es recomendable para el Sistema de Mediación Familiar de Chile. Para abordar esta pregunta general planteamos tres preguntas específicas: ¿ ¿cuáles son los beneficios que podría aportar la mediación en línea al Sistema de Mediación Familiar de Chile?; ¿cuáles son las dificultades que podría presentar dicha modalidad en el Sistema de Mediación Familiar de Chile?; ¿ es posible que el mediador pueda cumplir de manera adecuada sus labores en una mediación en línea, incluyendo la observancia de los principios del proceso? La hipótesis que planteamos es que la mediación en línea puede beneficiar a los usuarios y mediadores del Sistema de Mediación Familiar de Chile, y que no impide desempeñar de manera adecuada la labor mediadora o cumplir los principios del proceso. Nuestra investigación aportará antecedentes con rigor científico que servirán para conocer si la mediación en línea

5. Pascual Hernández Mergoldd, «La cultura de la paz, mediación a distancia en tiempos del covid-19», El Economista, 5 de mayo de 2020, disponible en https://bit.ly/3d3m7zg; «Primera mediación penitenciaria a distancia», Dirección Nacional de Mediación y Métodos Participativos de Resolución de Conflictos, 24 de octubre de 2017, disponible en https://bit.ly/2UTKuJv; «Mediación comunitaria online: Comenzó la prueba piloto», Ministerio de Justicia y Derechos Humanos de Argentina, 27 de agosto de 2019, disponible en https://bit.ly/3hAQCzT. 
puede beneficiar al Sistema de Mediación Familiar de Chile. Con estos resultados también se podrá dilucidar si es o no razonable que sólo se admita la concurrencia física a este Sistema.

Iniciamos este trabajo con una breve caracterización de la mediación en línea. Continuamos con una síntesis de los beneficios e inconvenientes que describe la literatura acerca de esta modalidad, y de las pautas de práctica internacionales dictadas para su implementación. Luego, describimos el Sistema de Mediación Familiar de Chile, los principios rectores del proceso, y revisamos el pronunciamiento de las autoridades de la Unidad de Mediación y Resolución Alternativa de Conflictos respecto de la mediación en línea. Por último, discutimos la información obtenida a partir de las preguntas de investigación y enunciamos nuestras conclusiones.

\section{La mediación en línea}

Como cualquier mediación, la mediación en línea es un método de resolución de conflictos no adversarial en el que participa un tercero imparcial denominado mediador para ayudar a las personas a resolver por sí mismas sus desavenencias (Peña, 2014: 11). La particularidad que presenta la mediación en línea es que se desarrolla por medio de tecnologías digitales que pueden o no estar conectadas a internet. Esto permite que los procesos de mediación se lleven a cabo sin necesidad de que los participantes se reúnan físicamente en un mismo espacio o tiempo (Alzate, 2008: 9, 13).

La mediación en línea puede ser sincrónica o asincrónica. El primer término se refiere a la situación en que el mediador y los usuarios pueden comunicarse e interactuar en tiempo real pese a estar en espacios diferentes, lo cual puede hacerse, por ejemplo, empleando software o aplicaciones móviles de videoconferencia o chat instantáneo. El segundo término describe el caso en que el mediador y los usuarios se comunican de manera diferida en el tiempo y desde espacios distintos, lo cual puede efectuarse, por ejemplo, por medio del uso de correo electrónico (Alzate, 2008: 11). Las tecnologías digitales determinan la forma en que los participantes pueden interactuar y comunicarse durante el proceso (Alzate y Vásquez, 2013: 33). Si los participantes emplean una plataforma básica de chat, probablemente sólo podrán comunicarse por medio de lenguaje escrito, y si optan por utilizar un teléfono de red fija, sólo a través de lenguaje verbal, y en ambos casos, de manera sincrónica. No obstante, en la actualidad, internet permite acceder a una amplia oferta de softwares y aplicaciones móviles con versiones gratuitas, que permiten comunicarse de forma sincrónica y asincrónica, e interactuar de manera simultánea o alternativa mediante texto, audio, imágenes y video, como Zoom, Whatsapp y Skype. 


\section{Beneficios y dificultades de la mediación en línea}

En la literatura se sugiere que la mediación en línea posee los siguientes beneficios:

- Si se desarrolla de manera asincrónica, ofrece la posibilidad de que cada participante pueda releer las respuestas que recibe y, al mismo tiempo, pensar con mayor detención sus propias comunicaciones. En consecuencia, favorece el proceso reflexivo de las personas en conflicto y evita que la comunicación se produzca en medio de estallidos emocionales (Alzate, 2008: 14; Lavi, 2015: 282).

- Si los participantes se comunican por medio de lenguaje verbal o escrito, permite crear un entorno que favorece la eliminación de sesgos vinculados a la apariencia, la raza, edad u otras características que podrían dificultar la comunicación o colaboración entre los participantes (Alzate, 2008: 14; Lavi, 2015: 288).

- Si los participantes se comunican por medio de lenguaje escrito, es más probable que el mediador pueda identificar los componentes agresivos en la comunicación y evitar la intimidación o violencia no verbal que pudiera manifestarse de forma implícita en posturas, gestos o movimientos (Lavi, 2015: 282, 285).

- Contribuye a desestabilizar las pautas de comunicación y relación establecidas previamente entre las personas en conflicto, incluyendo aquéllas que han originado o reforzado el desacuerdo en el que se encuentran (Alzate, 2008: 13).

- Permite ahorrar los costes de la convocatoria, es decir, el tiempo y energía que emplean los mediadores para conseguir que las personas en conflicto se reúnan en torno a una mesa, y el tiempo, dinero y energía que los usuarios emplean para concurrir a la sesión (Alzate, 2008: 14; Lavi, 2015: 288-289).

- Ofrece la posibilidad de que los mediadores y usuarios puedan elegir los espacios que les permitan sentirse más cómodos para participar del proceso de mediación (Alzate, 2008: 14).

- Permite eliminar las barreras geográficas, al hacer que la distancia física no sea un impedimento para acceder a la mediación (Alzate, 2008: 8; Wilson-Evered y otros, 2011: 128).

- Mediante la distancia física, puede contribuir a disminuir el desequilibrio de poder que antes puede haberse generado entre participantes que han experimentado situaciones violentas o de estrés significativo a causa del conflicto (Lavi, 2015: 281-284; Wilson-Evered y otros, 2011: 129). 
- Es más apropiada en los casos en que el temperamento de las personas es difícil de manejar, y previene las manifestaciones de violencia física entre las personas en conflicto o en contra de los mediadores (Alzate y Vásquez, 2013: 22; Lavi, 2015: 281).

- Si por cualquier razón la mediación presencial no es posible, pero las personas en conflicto desean ser parte del proceso, esta modalidad evita que queden impedidas de acceder al servicio (Alzate y Vásquez, 2013: 53).

En la literatura se sugiere que la mediación en línea presenta las siguientes dificultades:

- Requiere que los usuarios tengan acceso regular a tecnologías digitales e internet (Alzate y Vásquez, 2013: 74; Ebner, 2012: 368).

- Requiere que los usuarios posean familiaridad con el uso de tecnologías digitales (Alzate, 2008: 15; Ebner, 2012: 368).

- Requiere que los mediadores posean un alto nivel de conocimiento respecto del uso de internet y de las tecnologías digitales que se utilizarán en cada proceso (Ebner, 2012: 377).

- Si los participantes se comunican por medio de lenguaje escrito, es más difícil que se genere confianza y más fácil que se produzcan malas interpretaciones a partir de la lectura que hace cada persona (Alzate y Vásquez, 2013: 40; Lavi, 2015: 298). Además, el mediador quedará impedido de detectar las emociones que se expresan por medio de los elementos paralingüísticos o no verbales, y será más difícil que pueda intervenir y reducir la tensión de inmediato al no estar en contacto directo (Alzate, 2008: 12; Lavi, 2015: 299).

- Las herramientas de la mediación tradicional deben ser reexaminadas para determinar su idoneidad y aplicación en línea (Ebner, 2012: 376).

- Los procesos pueden ser afectados por intromisiones de terceros que pongan en riesgo el principio de confidencialidad y la seguridad de la información (Ebner y Zelesnikow, 2015: 157-158; Lavi, 2015: 296).

- Al situarse desde un espacio de confort, los participantes pueden mostrarse más desinhibidos y adoptar formas de comunicación e interacción agresivas o groseras (Lavi, 2015: 297). 


\section{Pautas de práctica internacionales para una adecuada implementación de la mediación en línea}

A continuación, presentamos una síntesis de las pautas que han sido publicadas en Australia, Estados Unidos, Reino Unido y Argentina para asegurar una adecuada implementación de la mediación en línea.

\section{Idoneidad de la mediación en línea}

Antes de iniciar un proceso de mediación en línea se recomienda que el mediador analice si dicha modalidad es idónea para el caso en concreto. Para lo anterior, en la guía de Mediate BC (2012: 11-12) se recomienda verificar si están presentes los siguientes criterios:

- Las partes tienen acceso al menos a un tipo de tecnología adecuada.

- Todas las partes acuerdan seguir la mediación en línea.

- Las partes poseen capacidades suficientes para hacer uso de la tecnología.

- Cualquier diferencia en las capacidades de las partes que usan la tecnología se puede gestionar de manera efectiva.

- Es poco probable que el uso de la tecnología impacte de manera negativa la relación.

- El uso de la tecnología no representa un alto riesgo en términos de seguridad o confidencialidad de la información de las partes.

- Si es necesario, se puede desarrollar un plan de seguridad para las partes a distancia.

- El uso de la tecnología no será un obstáculo para resolver la disputa.

- El uso de la tecnología es neutral u ofrece un beneficio para resolver la disputa.

Sin perjuicio de este análisis preliminar, los mediadores deben evaluar la idoneidad de la mediación en línea de manera continua, porque las circunstancias podrían variar en el transcurso del proceso (Mediate BC, 2012: 12).

\section{Elección de la tecnología digital}

Para elegir la tecnología digital más conveniente para el caso en concreto, se recomienda que el mediador evalúe:

- Cuáles son las tecnologías digitales que preferirían utilizar los usuarios. Se sugiere considerar con especial cuidado las preferencias del participante que 
posee un acceso más limitado a tecnologías o conectividad digital, o que tenga las habilidades tecnológicas o de comunicación más básicas, ya que esto puede ser un factor decisivo para evitar un desequilibrio de poder (Mediate BC, 2012: 13-14).

- Si las características de la tecnología podrían afectar de algún modo la relación entre los participantes o el desarrollo del proceso (Mediate BC, 2012: 11-12).

- Si posee los conocimientos y la experiencia necesaria para manejar en forma adecuada la tecnología digital escogida. En caso contrario, debe analizar si posee el tiempo suficiente para aprender a utilizar la tecnología y estar preparado al momento de la sesión respectiva. Además, debe evaluar cuál es el tipo de capacitación que requeriría un usuario que no está familiarizado con la tecnología y si es posible proveérsela (Mediate BC, 2012: 6, 8-9, 13).

- Cuáles son los gastos que se derivan del uso de la tecnología (Mediate BC, 2012: 7). El International Council for Online Dispute Resolution (ICODR) aconseja optar por tecnologías que puedan ser utilizadas gratuitamente por los usuarios. ${ }^{6}$

Además, el mediador debe verificar que:

- Tanto él como los usuarios posean una conexión de internet adecuada según las características de la tecnología que se pretende utilizar (Mediate BC, 2012: 7).7

- La tecnología digital ofrezca estándares elevados de seguridad, confidencialidad y privacidad de la información (Mediate BC, 2012: 6). ICODR sugiere utilizar plataformas con cifrado de extremo a extremo para asegurar la seguridad de los participantes. Además, se recomienda que la tecnología posea interfaces de fácil manejo para los usuarios y que el mediador pueda hacer un control adecuado sobre sus características (Mediate BC, 2012: 6). El Centre for Effective Dispute Resolution (CEDR) sugiere utilizar plataformas de videoconferencia como Zoom Pro, Webex, GoToMeeting o Skype for Business. ${ }^{8}$

\section{Protocolo de fallas tecnológicas}

Otro aspecto que se recomienda antes de iniciar una mediación en línea es que el mediador cuente con un protocolo de actuación específico frente a las fallas que puedan

6. «Video mediation guidelines», International Council for Online Dispute Resolution, disponible en https://bit.ly/3oKgre4.

7. «Video mediation guidelines».

8. «Mediator guide to online mediation», Centre for Effective Dispute Resolution, disponible en https://bit.ly/3fsluk6. 
presentar las tecnologías digitales. ${ }^{9}$ En la guía de Mediate BC (2012: 25) se sugiere que en dicho protocolo:

- Se establezca qué sucederá en los casos en que todos o alguno de los participantes tengan problemas de conectividad, o se presenten fallos en la tecnología digital que impidan su uso o limiten sus funcionalidades.

- Se determine cuáles serán las vías alternativas de contacto que utilizarán los participantes para comunicar los problemas de conectividad o fallos de la tecnología digital.

- Se defina al menos una tecnología alternativa que permita que todos los usuarios puedan comunicarse de manera equivalente a lo inicialmente acordado.

- Se consideren las vías de acción que resulten más cómodas para los usuarios del proceso. Para ello, si es posible, se sugiere identificarlas en conjunto con ellos.

\section{Gestión de la confidencialidad y seguridad}

Dada la naturaleza de las tecnologías digitales, el mediador no puede garantizar que todas las comunicaciones sean confidenciales o controlar por completo dónde o cómo se puede recopilar, almacenar o acceder a parte de la información del proceso. Sin embargo, la gestión de riesgos es esencial y es deber del mediador hacer todos los esfuerzos razonablemente posibles para proteger la privacidad de los participantes y la confidencialidad del proceso (Mediate BC, 2012: 16). Para lo anterior, se recomienda que el mediador:

- Se informe con cuidado sobre las características de seguridad de las tecnologías digitales que se pretende utilizar, de modo que pueda asegurarse de que sean suficientes para los fines de la mediación. Es imperativo evitar las plataformas digitales en las que cualquier información sobre la mediación pueda hacerse pública. Se sugiere que el mediador tenga en cuenta la importancia del cifrado de información, de la autenticación y de las contraseñas de seguridad. Si se utilizan plataformas de videoconferencia web, por ejemplo, éstas deberían proporcionar al mediador la posibilidad de establecer controles de acceso, una lista visible de participantes y medidas de autenticación para los asistentes. ICODR recomienda utilizar plataformas de videoconferencia con cifrado de extremo a extremo y no usar aplicaciones o software que requieran que los usuarios compartan información personal innecesaria, como datos sobre su ubicación.

9. Centre for Effective Dispute Resolution, «Mediator guide...». 
- Se asegure de que sus sistemas informáticos estén protegidos en todo momento contra virus, gusanos, troyanos y otras amenazas a la integridad y seguridad de la información. Se recomienda cifrar la información personal que esté almacenada en dispositivos vulnerables, como computadoras, portátiles o unidades USB (Mediate BC, 2012: 18).

- Se asegure de que el acceso remoto a los datos en una computadora de oficina, red organizacional o servicio de nube solo esté permitido desde un sitio web confiable o mediante el uso de contraseñas (Mediate BC, 2012: 18).

- Antes de deshacerse de la computadora o dispositivo que emplea para la mediación, debe asegurarse de que los datos que contiene sean eliminados o que el medio que los almacena se destruya. Se recomienda evitar el uso de computadoras públicas y sistemas inalámbricos públicos para transmitir o acceder a información confidencial (Mediate BC, 2012: 18).

- Asesore a las partes sobre los riesgos de confidencialidad inherentes al uso de la tecnología y las formas para minimizar esos riesgos (Mediate BC, 2012: 18).

- Solicite a las partes que confirmen su identidad antes de comenzar una mediación (Mediate BC, 2012: 19).

- Solicite a las partes que acepten no tener a otra persona en la sala o dentro de una distancia cercana cuando participen en la mediación, a menos que el permiso sea obtenido del mediador y la otra parte. Se recomienda preguntar siempre a cada participante si hay alguien cerca o dentro de la sala en la que se encuentra antes de iniciar un proceso. También es esencial que las partes entiendan la necesidad de tomar precauciones para evitar que sus hijos escuchen las discusiones (Mediate BC, 2012: 18). En la guía dictada por el Ministerio de Justicia y Derechos Humanos de Argentina se establece que al inicio de la audiencia el mediador debe enfatizar la confidencialidad del procedimiento y los participantes deben manifestar que no se encuentran presentes personas ajenas al procedimiento, ni observando ni escuchando por cualquier medio. ${ }^{10}$

- Considere el hecho de que algunas partes pueden verse tentadas a grabar las discusiones mantenidas durante la mediación en línea. Si bien puede que no sea posible evitar que una parte determinada registre discusiones, existen estrategias para minimizar la posibilidad de que ocurra. Por ejemplo, si se utiliza una plataforma de conferencia web que permite grabar reuniones, el mediador debe asegurarse de que las partes no puedan acceder a esta función (Media-

10. «Guía para la realización de mediaciones a distancia», Ministerio de Justicia y Derechos Humanos de la República Argentina, disponible en https://bit.ly/2YHE2pS. 
te BC, 2012: 19). El ICODR recomienda incluir en el contrato de mediación una cláusula en la que los participantes se comprometen a no tomar registro del proceso, y que el mediador recuerde este compromiso antes de iniciar el proceso. En la guía dictada por el Ministerio de Justicia y Derechos Humanos de Argentina, se establece que al inicio de la audiencia, el mediador deberá informar sobre la prohibición de grabar y reproducir el proceso por cualquier medio, y que los participantes deben comprometerse a no transgredir dichas prohibiciones.

- Obtener la asistencia de un experto en tecnologías y conectividad digital a la hora de tratar cualquier área de incertidumbre técnica (Mediate BC, 2012: 16).

\section{Preparación del entorno de mediación}

Si se desea utilizar una plataforma de videoconferencia, CEDR recomienda al mediador que tome medidas respecto del entorno que mostrará a los participantes. Se sugiere contar con una pieza iluminada, en la que se aprecie limpieza, orden y elementos similares a los que se utilizarían en una oficina de mediación.

En la guía de Mediate BC (2012: 19; 35-37) se indica que los usuarios que desean participar de una mediación en línea deben disponer de un espacio tranquilo, donde no exista ruido ambiental excesivo, la intromisión de terceros, o la presencia de elementos distractores que impidan o dificulten la concentración requerida para el proceso. Por ello, se recomienda que durante la premediación el mediador se encargue de que cada usuario entienda la importancia de preparar su entorno de mediación y si es posible, los oriente acerca de cómo alcanzar los estándares requeridos.

\section{Información previa al proceso de mediación en línea}

Se recomienda que durante la premediación, el mediador explique a los participantes cómo la mediación se verá afectada por la distancia y el uso de tecnologías digitales, y les indique cuáles son los compromisos que impone el uso de la tecnología en el proceso (Mediate BC, 2012: 21) Por lo anterior, es importante que el mediador converse con los participantes sobre:

- Cuál será la duración del proceso y la modalidad de las sesiones (Mediate BC, 2012: 35). CEDR recomienda que cada sesión tenga un límite máximo de 60 minutos.

- Cuál será la tecnología digital que se utilizará para llevar a cabo el proceso y el contenido del plan de fallas de tecnológicas. Se sugiere que el mediador explique con naturalidad a los participantes el hecho de que las tecnologías no son perfectas y el fin de establecer el plan de fallas (Mediate BC, 2012: 35, 40). 
- Cuáles serán las reglas de uso de cada tecnología y las reglas básicas de comunicación que regirán durante el proceso (Mediate BC, 2012: 35).

- Cuáles son las condiciones de seguridad que ofrece la tecnología digital y el contenido del plan de gestión de seguridad (Mediate BC, 2012: 57).

- Cuáles son los requisitos que debe poseer el entorno desde el cual se situará cada participante durante el proceso de mediación. Se recomienda explicitar que se requiere un ambiente tranquilo, sin intromisiones, distracciones o ruidos excesivos (Mediate BC, 2012: 37, 57).

- De qué forma se acreditará la identidad de cada participante (Mediate BC, 2012: 50).

- De qué manera serán firmados los documentos (Mediate BC, 2012: 50) - por ejemplo, el acuerdo, en caso de haberlo- y como serán enviados al mediador. Sobre este punto, el protocolo del Poder Judicial de la Provincia de San Luis de Argentina indica que se procederá a grabar el momento en que los participantes manifiesten su voluntad de arribar a un acuerdo total o parcial, lo cual deberá ser informado por el mediador antes de iniciar el proceso. ${ }^{11}$ En el protocolo del Poder Judicial de la Provincia de Neuquén de Argentina ${ }^{12}$ se establece que en caso de arribarse a un acuerdo de partes, el mediador deberá redactar el texto del acta acuerdo bajo la modalidad de texto único que trasmitirá vía correo electrónico o Whatsapp a los participantes, para obtener y garantizar su expresa conformidad con éste, y una vez obtenida la conformidad de todas las partes, el mediador deberá firmar digitalmente el acta y notificarla a través de notificación electrónica a los letrados patrocinantes y al juzgado interviniente.

\section{Recomendaciones adicionales para el mediador en línea}

Se recomienda que el mediador:

- Evalúe en forma periódica el funcionamiento de las tecnologías digitales y del plan de fallas tecnológicas (Mediate BC, 2012: 25, 39).

- Haga un esfuerzo adicional para la creación de confianza. Ello implica trabajar sobre la presentación personal, el entorno de trabajo y analizar con cuidado el uso de los elementos paralingüísticos, no verbales y el nivel de escucha y de atención que se mantiene y demuestra en el proceso (Mediate BC, 2012: 23-24, 47).

11. «Pautas de trabajo para la realización de audiencias bajo la modalidad virtual», Poder Judicial de la Provincia de San Luis, disponible en https://bit.ly/2YHE6WE.

12. «Se amplían las mediaciones y conciliaciones a distancia durante la cuarentena en todo el país», Centro de Información Judicial, 21 de abril de 2020, disponible en https://bit.ly/2ULIL8V. 
- Mantener las emociones a un nivel manejable puede ser un desafío en la mediación en línea, por lo que es particularmente importante que consulte en forma regular a las partes qué piensan en lugar de qué sienten (Mediate BC, 2012: 44).

- Si se utiliza videoconferencia, se recomienda que sitúe la mirada hacia la pantalla de los demás participantes y no en la propia imagen. En estos casos será importante que la cámara esté ubicada de manera frontal para que los usuarios puedan ver su rostro y hacer contacto visual. También se sugiere que el mediador eduque a los usuarios sobre la manera de situar su cámara (Mediate BC, 2012: 24, 36). ${ }^{13}$

- Aproveche al máximo las fortalezas de las tecnologías: si se utilizan tecnologías con múltiples funciones, por ejemplo, se sugiere emplear estas características de varias maneras para enriquecer el proceso de mediación (Mediate BC, 2012: 42).

- No asistir a otras tareas mientras se media a través de videoconferencia, como responder correos electrónicos o desviar la mirada hacia el teléfono celular (Mediate BC, 2012: 47).

- Adoptar medidas adicionales para confirmar que los participantes entienden los términos del acuerdo alcanzado en el proceso de mediación (Mediate BC, 2012: 49).

\section{El Sistema de Mediación Familiar de Chile}

El Sistema Nacional de Mediación Familiar depende de la División Judicial del Ministerio de Justicia y Derechos Humanos. Este sistema se implementó anexo a los Tribunales de Familia para ampliar y garantizar el acceso de los ciudadanos a la justicia de familia, hacer más heterogénea la tutela de derechos ofrecida por el Estado, y procurar soluciones pacificadoras a favor de la armonía familiar. ${ }^{14}$ Por mandato del artículo 112 de la Ley 19.968, el Sistema cuenta con un registro público de mediadores familiares. Este registro está integrado por mediadores licitados y privados. Los primeros son contratados por la División Judicial para prestar servicios de mediación en las materias de mediación previa y obligatoria y sin costo para los usuarios del país. Los segundos están habilitados para prestar servicios particulares de mediación en las materias de mediación previa y obligatoria, o en otras que no estén prohibidas por la ley. $\mathrm{Al} 7$ de enero de 2020, este registro estaba integrado por 3.920 mediadores. ${ }^{15}$

13. Centre for Effective Dispute Resolution, «Mediator guide...».

14. «Historia de la Ley 19.968», Biblioteca del Congreso Nacional de Chile, 30 de agosto de 2004, pp. 33, 755, 1.039, disponible en https://bit.ly/2Y2WGJA.

15. Dato obtenido mediante solicitud de información ingresada el 16 de diciembre de 2019 al Portal 


\section{Los principios rectores de la mediación familiar chilena}

La regulación legal de la mediación familiar se contiene en el título 5 de la Ley 19.968, que comprende desde el artículo 103 al 114 inclusive. El artículo 105 consagra los principios rectores del proceso, a saber: los principios de igualdad, de voluntariedad, de confidencialidad, de imparcialidad, de interés superior del niño y de opiniones de terceros. En virtud del principio de igualdad, el mediador debe cerciorarse de que los participantes se encuentren en igualdad de condiciones para adoptar acuerdos; si no fuese así, su deber consiste en proponer o adoptar las medidas necesarias para que se obtenga ese equilibrio. Si lo anterior no es posible, el mediador debe declarar terminada la mediación (artículo 105, letra a). El principio de voluntariedad faculta a los participantes para retirarse de la mediación en cualquier momento. Si en la primera sesión, o en cualquier otro momento del proceso, alguno de los participantes manifiesta su intención de no seguir adelante con la mediación, ésta se tendrá por terminada (artículo 105, letra b). El principio de confidencialidad obliga al mediador a guardar reserva de lo escuchado o visto durante el proceso de mediación y lo ampara bajo el secreto profesional. La violación de dicha reserva es sancionada penalmente. Además, este principio prohíbe que lo dicho por cualquiera de los participantes durante el desarrollo de la mediación pueda invocarse en un subsiguiente procedimiento judicial. Con todo, el mediador queda exento del deber de confidencialidad en aquellos casos en que tome conocimiento de la existencia de situaciones de maltrato o abuso en contra de niños, adolescentes o personas con discapacidad (artículo 105, letra c). El principio de imparcialidad impone al mediador el deber de abstenerse de promover actuaciones que comprometan dicha condición. Si tal imparcialidad se viere afectada por cualquier causa, el mediador deberá rechazar el caso, justificándose ante el juzgado que corresponda. Los usuarios también podrán solicitar al juzgado la designación de un nuevo mediador, cuando justifiquen que la imparcialidad del inicialmente designado se encuentra comprometida (artículo 105, letra d). El principio del interés superior del niño obliga al mediador a velar para que se tome en consideración dicho interés durante el curso del proceso (artículo 105, letra e). El principio de opiniones de terceros obliga al mediador a considerar las opiniones de terceros que no hubieren sido citados, a quienes también podrá citar (artículo 105, letra f).

de Transparencia del Estado para el organismo Subsecretaría de Justicia del Ministerio de Justicia y Derechos Humanos de Chile. 


\section{Pronunciamiento de las autoridades de la Unidad de Mediación y Resolución Alternativa de Conflictos respecto de la mediación en línea}

El 25 de marzo de 2020, las autoridades de la Unidad de Mediación y Resolución Alternativa de Conflictos de la División Judicial del Ministerio de Justicia y Derechos Humanos de Chile emitieron un pronunciamiento en el que descartan la posibilidad de que en el contexto de la pandemia por covid-19 pudieran hacerse mediaciones en línea. Los fundamentos de dicha prohibición fueron los siguientes.

Respecto del marco legal, las autoridades de la Unidad indicaron:

No es posible considerar una mediación a distancia utilizando medios de comunicación electrónica como la telefónica, videoconferencia u otro similar, toda vez que el verbo normativo «deberán» le da el carácter de imperativo al artículo 108, estableciendo requisitos para que el acto jurídico pueda llevarse a efecto, como lo es la concurrencia personal de las personas involucradas en el conflicto, «quienes deberán comparecer personalmente». ${ }^{16}$

Además, agregaron que,

no siendo necesario que las partes y el mediador utilicen tiempo y recursos para reunirse en el mismo espacio físico al mismo tiempo, podría darse el caso de que las partes realicen un proceso de mediación sin los estándares mínimos requeridos por el Ministerio de Justicia y Derechos Humanos, o sin dar estricto cumplimiento a los principios establecidos por la ley como son la confidencialidad, voluntariedad y otros, y lo que es más grave, aquellos casos relacionados con violencia, intimidación o desequilibrio de poder entre las partes, expresamente previstos por el legislador, serían prácticamente indetectables, no pudiendo el mediador utilizar las herramientas que le permitieran, en estos casos, frustrar el proceso, en resguardo, precisamente de aquella parte que fue víctima de un hecho de esa naturaleza. ${ }^{17}$

Respecto de la labor mediadora, las autoridades indicaron:

La asunción de los procesos de mediación está pensada para la interacción presencial y sólo en estas condiciones pueden ser aplicadas las técnicas de mediación y obtener así los beneficios de la institución. Si existe la posibilidad de que cualquiera de los participantes pueda utilizar el registro electrónico de lo que sucedió o se dijo en la sesión, para fines ajenos al proceso, la mediación pierde su carácter de confidencial y esta distancia no permite al mediador tener control de la señalada confidencialidad del proceso de mediación. De la misma forma, resulta imposible controlar la presencia de personas ajenas al proceso de mediación, tales como abogados de las partes, familiares, etcétera. La mediación a distancia no permite al mediador equilibrar los

16. Unidad de Mediación y Resolución Alternativa de Conflictos, «Pronunciamiento...», 2.

17. Unidad de Mediación y Resolución Alternativa de Conflictos, «Pronunciamiento...», 3. 
poderes de los participantes, pues pierde parte de su capacidad para interrumpir a una persona que reacciona mal, que evade, o que insulta, no le permite interrumpir oportunamente la sesión para convocar a reuniones privadas, etcétera. Existe el riesgo de que el conflicto escale si el mediador no tiene la capacidad de intervenir directamente, ya sea conteniendo, calmando la tensión inmediatamente mediante el contacto directo. El uso de tecnologías a distancia puede llevar a distorsiones y malas interpretaciones dentro del proceso de mediación, no pudiendo garantizar un estándar mínimo de calidad en la prestación del servicio. ${ }^{18}$

Por último, respecto de factores geográficos, sociales y económicos, las autoridades indicaron que

en algunas zonas de Chile existen dificultades de comunicación, o no existen los medios tecnológicos o económicos que permitan a los usuarios acceder a ellos. Sin perjuicio de lo anterior, y aun pudiendo existir esos medios, la mediación a distancia no permite preservar la integridad de los procesos de mediación presenciales, ni el cumplimiento irrestricto de sus principios, por ende, permitirla significaría atentar en contra de la institución, tal como fue concebida por el legislador. ${ }^{19}$

\section{Discusión}

En el contexto de la pandemia por covid-19, las autoridades de la Unidad de Mediación y Resolución Alternativa de Conflictos descartaron que fuera posible dar continuidad al servicio mediante la mediación en línea. Como fundamento inmediato de esta prohibición se mencionó la incompatibilidad de dicha modalidad con la concurrencia personal que exige el artículo 108 de la Ley 19.968. Además, las autoridades de la Unidad indicaron que la mediación familiar en línea no resultaba conveniente para Chile por factores geográficos, sociales y económicos. Por último, para desestimar en forma terminante la posibilidad de implementar esta modalidad, las autoridades indicaron que solo la mediación presencial permitía un desempeño adecuado de la labor mediadora y cumplir los principios del proceso. Sin embargo, en este pronunciamiento las autoridades de la Unidad no ofrecieron datos o estudios que permitieran respaldar sus aseveraciones.

Por lo anterior, la pregunta general que nos planteamos en esta investigación fue si era correcto afirmar en forma categórica que la mediación en línea no fuese recomendable para el Sistema de Mediación Familiar de Chile. Para responder esta interrogante, planteamos tres preguntas específicas que a continuación pasamos a discutir.

18. Unidad de Mediación y Resolución Alternativa de Conflictos, «Pronunciamiento...», 4. 19. Unidad de Mediación y Resolución Alternativa de Conflictos, «Pronunciamiento...», 6. 


\section{¿Cuáles son los beneficios que podría aportar la mediación en línea al Sistema de Mediación Familiar de Chile?}

La implementación de la mediación en línea permitiría ampliar las vías de acceso a la justicia familiar colaborativa, lo cual coincide con los fines que el legislador chileno se propuso alcanzar con el Sistema Nacional de Mediación Familiar. En concreto, a través de la mediación en línea podría evitarse que los ciudadanos que quieran acceder a una mediación familiar queden impedidos de hacerlo por el hecho de no poder asistir físicamente a los centros de mediación. Como se señala en la literatura, este obstáculo puede surgir por la distancia geográfica que separa a las personas que desean participar del proceso. Este factor resulta especialmente trascendente para Chile, ya que cuenta con $4.270 \mathrm{~km}$ de largo y altas tasas de migración interna (Rodríguez, 2019: 19-30). La distancia geográfica puede aumentar de manera significativa los gastos de convocatoria de una mediación, pues para reunirse en torno a una mesa al menos uno de los participantes debe asumir gastos extraordinarios de transporte, alimentación o alojamiento. El problema radica en que no todas las familias pueden asumir tales gastos con el fin de participar de una mediación; sin embargo, una mediación en línea podría ser una alternativa más económica y accesible. También se debe considerar que la distancia geográfica puede acompañarse de otros factores que podrían obstaculizar la asistencia presencial a las sesiones de mediación, como la falta de tiempo o de transporte para desplazarse hacia un lugar lejano. Estas dificultades también podrían ser superadas por medio de la mediación en línea.

Los obstáculos para asistir a un centro de mediación también pueden presentarse a los participantes que se ubican en una misma zona geográfica cuando, por ejemplo, alguno de ellos está recluido en un recinto penitenciario, padece una enfermedad inmunodepresora o está afectado por una discapacidad física que genera graves molestias de desplazamiento. En estos casos, la mediación en línea también podría ser útil para evitar que dichas personas deban sortear obstáculos significativos para acceder al servicio. Otra situación posible es que los usuarios no puedan asistir a los centros de mediación a causa de la declaración de estado de excepción constitucional, como ocurre con la pandemia de covid-19. En estos casos, la mediación en línea sería una alternativa para dar continuidad al servicio. En particular, en el contexto de la emergencia por covid-19, la mediación en línea permitiría reactivar el Sistema Nacional de Mediación Familiar sin que implique un riesgo para la salud de la población. Esto resulta en especial importante si se considera el tiempo que podría tardar en estar disponible una vacuna para la enfermedad en Chile. Mientras esto no ocurra, aun cuando se retome el funcionamiento presencial de los centros de mediación, existirá un alto porcentaje de población vulnerable, como enfermos crónicos, embarazadas o adultos mayores, que no podrían asistir a una mediación por el alto riesgo que significaría exponerse al contagio. Por lo tanto, si en dichas circunstancias a la población 
de riesgo no se le proveyera una alternativa segura para mediar, indirectamente se les estaría negando el acceso a la justicia colaborativa.

Otro beneficio que podría ofrecer la mediación en línea sería posibilitar el acceso directo de los usuarios a las sesiones de mediación. Con esto se evitaría que los participantes deban esperar su respectivo llamado a ingresar a mediación en una sala de espera pública. Esta funcionalidad podría ser útil, por ejemplo, para las personas que cuentan con limitada disponibilidad de tiempo, para quienes temen encontrarse con los acompañantes del otro participante, o para quienes presentan alguna dificultad en el manejo de la ansiedad social. En el contexto de la pandemia de covid-19 resulta evidente la importancia de evitar aglomeraciones.

También creemos que si los usuarios del Sistema Nacional de Mediación Familiar tuvieran la posibilidad de elegir entre la modalidad presencial o en línea, se estaría potenciando desde un inicio su protagonismo y el principio de voluntariedad, ya que éstos no solo tendrían la opción de decidir si desean o no participar de una mediación, sino que además podrían escoger la modalidad que más les acomode para hacer efectiva su participación. Contemplar esta alternativa podría ser especialmente pertinente considerando la diversidad de familias que coexisten en el país, pues como ya señalamos, no todas las personas viven realidades en las que concurrir a una mediación sea viable. También se debe considerar la existencia de familias formadas por nativos digitales o familias tradicionales que han optado o debido adecuarse a la digitalización y que requieren soluciones acordes a sus estilos de vida. La digitalización es una realidad que se impone en los distintos ámbitos de la vida en sociedad, y esto se ha visto maximizado a propósito de la emergencia sanitaria por covid-19. Por lo tanto, creemos que hoy más que nunca existe un contexto favorable en términos de conocimiento y familiaridad con las tecnologías digitales que se expresa en personas de todas las edades y que podría favorecer la implementación de la mediación en línea.

Uno de los beneficios de la mediación en línea que se indica en la literatura es que permite que las personas puedan situarse desde un espacio más cómodo o conocido, lo cual creemos podría fomentar la participación de los usuarios que por primera vez son convocados a una mediación y que sienten temor de asistir por la novedad del proceso. Además, creemos que este factor puede contribuir a que los usuarios expresen con más facilidad sus sentimientos, intereses o pensamientos. Los demás beneficios que describimos al revisar la literatura, como los relativos a la comunicación, el equilibrio del poder y el resguardo físico, también serían útiles para los usuarios del Sistema Nacional de Mediación Familiar.

La emergencia sanitaria por covid-19 ha dificultado y en muchos casos impedido la asistencia de mediadores y usuarios a los procesos de mediación. Este problema ha afectado en especial a los mediadores privados, quienes al no poder desarrollar sus servicios particulares, no perciben ingresos económicos. En la actualidad, pese a que los mediadores licitados no se encuentran ejerciendo mediaciones presenciales, éstos 
continúan siendo remunerados por el Estado. No obstante, es probable que el Estado reduzca los recursos públicos invertidos si las circunstancias que obligan a detener los servicios presenciales se extienden de manera prolongada en el tiempo, ya que no podrían prestarse los servicios comprometidos a la ciudadanía. La mediación en línea podría favorecer a los miles de mediadores que integran el Sistema Nacional de Mediación Familiar, ya que con ella no se verían obligados a dejar de trabajar cuando no puedan reunirse físicamente con los usuarios en los centros de mediación. Por ello, contar con la mediación en línea sería relevante para el universo de mediadores de Chile.

\section{¿Cuáles son las dificultades que podría presentar la mediación en línea en el Sistema de Mediación Familiar de Chile?}

La primera dificultad que atravesaría la mediación en línea se encuentra en la interpretación de las autoridades de la Unidad, debido a que consideran que esta modalidad no es compatible con la concurrencia personal que exige el artículo 108 de la Ley 19.968. Esta interpretación coincide con el significado que se atribuye a la comparecencia personal en la tradición jurídica, es decir, aquélla que se produce cuando una persona (el citado a comparecer) se presenta físicamente ante el juez. Por lo tanto, en el contexto de la mediación este requisito sólo se cumpliría si todos los usuarios citados concurren físicamente ante el mediador, a quien se le exige desempeñar su labor en un centro de mediación. De esta manera, al ser la Ley 19.968 una norma de derecho de familia y de orden público, se requeriría reformar el artículo 108 para poder implementar la mediación en línea. Sin embargo, creemos que esta dificultad podría solucionarse sin necesidad de una reforma legal, si se admitiera que en la época actual es posible concebir que la concurrencia personal al proceso de mediación no solo es posible por medio de la presencia física. Esto implicaría ampliar la noción de concurrencia personal en la mediación chilena, pasando de un concepto que se sujeta a las limitaciones de la naturaleza humana, en el que solo se considera posible la concurrencia personal física, a un concepto que atienda a la funcionalidad de dicha concurrencia. Es decir, si la finalidad de la concurrencia personal de los usuarios es que no exista la posibilidad de representación, consideramos que esto bien podría lograrse por medio de una videoconferencia, en que la comunicación es sincrónica y la identidad personal de los participantes está a la vista y puede ser contrastada con los respectivos documentos de identidad.

Por otro lado, la mediación en línea también podría dificultar el proceso de firma de las actas de acuerdo en los términos que indica el artículo 111 de la Ley 19.968. El mencionado artículo obliga a los participantes y al mediador a estampar sus firmas en la copia de acuerdo que corresponde a cada parte. Lo anterior no sería un problema si las partes contaran con firma electrónica, pero lo cierto es que este servicio 
todavía resulta inusual dentro de la población chilena. Sin embargo, en este punto, creemos que al igual como mencionamos en el párrafo anterior, optar por un criterio de funcionalidad podría ser apropiado para superar las limitaciones de la naturaleza humana. Es decir, si la firma de documentos en la mediación chilena tiene por fin acreditar que una persona determinada hizo una manifestación expresa de voluntad, creemos que esto bien podría lograrse mediante la grabación del momento en que las personas manifiestan su conformidad o rechazo respecto del contenido de los documentos, como se aplica en los centros de mediación de la provincia de San Luis, Argentina. No obstante, si la grabación fuera inviable, esta manifestación también podría hacerse por otros medios, por ejemplo, mediante una casilla de verificación digital en la que las personas pudieran manifestar el acuerdo o desacuerdo con respecto de los documentos que se les presentan, como ya se aplica en la contratación electrónica.

Otra dificultad que es mencionada por las autoridades de la Unidad se refiere al escaso acceso a tecnologías, conocimientos o conectividad digital que existe en determinadas zonas del país. Sin embargo, respecto de esta situación, cabe señalar que, según cifras publicadas por la Subsecretaria de Telecomunicaciones, durante el primer semestre de 2019 se mostró un alza significativa del uso de internet y de teléfonos móviles en Chile. En particular, en el primer semestre de 2019 la penetración de internet móvil alcanzó al 97,5\% de los habitantes de Chile. ${ }^{20}$ Además, hay que considerar que en el año 2022 entrará en operación el proyecto denominado «Fibra Óptica Nacional», que considera un subsidio estatal histórico de más de $\$ 86.000$ millones y que contempla el despliegue de $10.000 \mathrm{~km}$ de fibra óptica. Este proyecto beneficiara a 186 comunas desde la región de Arica y Parinacota hasta la región de Los Lagos. ${ }^{21}$ Por lo tanto, los datos demuestran que en Chile existen altos niveles de acceso a tecnologías, conocimiento y conectividad digital, y que se están adoptando medidas para mejorar aún más la conectividad digital del territorio nacional. Además, las circunstancias relacionadas al fenómeno covid-19 han hecho que la sociedad dependa y se familiarice con las tecnologías digitales. Todo lo anterior nos permite sostener que existen las condiciones técnicas y de conocimientos digitales en la sociedad para una implementación expedita de la mediación familiar en línea en Chile.

Sin duda la situación más compleja para la implementación de la mediación en línea sería en el marco de la pandemia por covid-19, debido a la alta demanda que

20. «Usuarios de internet móvil consumen $10 \mathrm{~GB}$ en promedio al mes y número de teléfonos móviles sube a 26 millones», Subsecretaría de Telecomunicaciones de Chile, 18 de octubre de 2019, disponible en https://bit.ly/3hvsWwS.

21. «Informe resultado del proceso de evaluación Subtel y adjudicación del Consejo de Desarrollo de las Telecomunicaciones del concurso público "Fibra Óptica Nacional”», Subsecretaría de Telecomunicaciones de Chile, disponible en https://bit.ly/3fEmisR. 
recibirá este servicio en un corto periodo. En estas circunstancias, el Estado deberá garantizar el acceso igualitario. Para facilitar el acceso a quienes no dispongan de conexión a internet, el Ministerio de Justicia y Derechos Humanos podría proveer acceso a los usuarios de mediación licitada a través de la contratación del servicio a empresas proveedoras de internet móvil. A modo ilustrativo, en una videoconferencia con tres dispositivos conectados, la cantidad de datos de audio y video transmitidos por persona es de alrededor de 2,7 GB, lo cual tiene un costo aproximado de $\$ 6.061$ pesos chilenos..$^{22}$ Esta cifra es significativamente menor al gasto público que podría derivarse de la judicialización de la misma controversia, y permitiría reactivar un servicio que hasta el momento el Estado financia, pero que no puede ser prestado a la comunidad. Luego, una vez superada la crisis sanitaria, una alternativa para asegurar que más personas puedan acceder a la mediación en línea, por ejemplo, podría ser facilitar a los usuarios el acceso desde los mismos centros de mediación licitados. De esta manera, una persona podría participar de una mediación en línea desde su casa con otra situada desde el centro de mediación en una misma o diferente zona geográfica.

Para contribuir a disminuir los riesgos de ciberataques que se describen en la literatura y que podrían afectar la confidencialidad y privacidad de la información, creemos que sería conveniente que el Sistema Nacional de Mediación opte por utilizar una aplicación o software de diseño propio, para que pueda ser programado específicamente según los estándares de seguridad que requiere la mediación. Lo anterior, debido a que las plataformas digitales que se comercializan no cuentan con los estándares más altos de seguridad, pues por lo general no fueron diseñadas para el tratamiento o tráfico de datos personales o sensibles.

Por otro lado, con respecto de las dificultades que podría traer aparejada la utilización de tecnologías digitales que solo permiten comunicarse por medio de voz o texto, éstas podrían ser abordadas mediante el uso de software o aplicaciones de videoconferencia, ya que de esta manera la comunicación sería sincrónica y no se prescindiría de elementos paralingüísticos o no verbales. Los demás obstáculos que se describen en la literatura, como la posibilidad de que los usuarios adopten formas de comunicación inapropiadas o que se vean tentados a grabar el proceso, creemos que como muestra la experiencia internacional, podrían gestionarse de manera adecuada por medio del establecimiento de protocolos que contengan normas claras de participación y de seguridad. En la actualidad, el Sistema Nacional de Mediación Familiar establece reglas básicas de participación para los usuarios de los procesos de mediación familiar presencial. Estas reglas son parte integrante de un consentimiento informado que los usuarios deben leer y firmar voluntariamente para participar de una mediación. Dentro de estas normas se encuentran, por ejemplo, el deber de evitar

22. Subsecretaría de Telecomunicaciones, «Usuarios...». 
agresiones verbales y de respetar los turnos de habla..$^{23} \mathrm{Si}$ los usuarios no respetan estas normas, el mediador puede frustrar el proceso. En la práctica, estas normas también son reiteradas verbalmente por el mediador en el discurso informativo al inicio. De igual forma, consideramos que se debiese informar a los usuarios sobre las normas de participación y seguridad que podrían establecerse para la mediación en línea. Así, podrían conocer los antecedentes mínimos para ejercer el principio de voluntariedad y decidir si desean o no participar de una mediación en línea. Asimismo, como indica la experiencia internacional, también sería conveniente informar a los usuarios sobre estas normas en la etapa de premediación, ya que así ellos podrían evaluar si la mediación en línea es la modalidad que necesitan para resolver su controversia antes de iniciar cualquier tipo de trámite o solicitud formal.

¿Es posible que el mediador pueda cumplir de manera adecuada sus labores en un proceso de mediación en línea, incluyendo la observancia de los principios de la mediación familiar?

Pese a que las autoridades de la Unidad señalan que solo la mediación presencial permite aplicar las técnicas de mediación y obtener los beneficios de la institución, los antecedentes analizados muestran que aquello no es efectivo, pues ni siquiera las desventajas que se señalan en la literatura sobre esta modalidad permiten arribar a dichas conclusiones. Por el contrario, la literatura muestra que la mediación en línea permite llevar a cabo en forma integral el proceso y aportar sus respectivos beneficios a los participantes.

Con respecto a la aprensión de las autoridades de la Unidad acerca de que los usuarios de una mediación en línea puedan tomar registro del proceso o estar acompañados de personas ajenas a éste, como ya hemos señalado, existe evidencia de que estos riesgos pueden ser contrarrestados de manera efectiva mediante el establecimiento de normas claras de participación. También reiteramos que sería conveniente informar a los usuarios acerca de este tipo de normas en la premediación, en el discurso inicial que efectúa el mediador y en el consentimiento informado que ellos deben leer y firmar voluntariamente antes del proceso. Además, se debe considerar que el artículo 105 letra c), inciso segundo de la Ley 19.968, al regular el principio de confidencialidad, señala que «nada de lo dicho por cualquiera de los participantes durante el desarrollo de la mediación podrá invocarse en el subsiguiente procedimiento judicial, en caso de haberlo». De esta manera, la propia Ley se encarga de prevenir uno de los principales riesgos asociados a la divulgación de información por parte de los usuarios.

23. «Consentimiento informado», Mediación de Conflictos Familiares, disponible en https://bit. ly/2C $7 \mathrm{Zqgl}$. 
La experiencia internacional también demuestra que no es efectivo que mediante una mediación en línea el mediador pierda el control del proceso, o que no pueda equilibrar los poderes de los participantes o evitar malas interpretaciones, sino que, por el contrario, se muestra que esta modalidad ofrece opciones particularmente útiles para disminuir dichos riesgos. Para lograrlo, como se señala en las pautas internacionales, es importante que la tecnología permita que el mediador sea capaz de controlar en forma adecuada sus características, de modo de poder intervenir en los casos en que detecte violencia o desequilibrios de poder que hagan necesaria una sesión individual o la frustración del proceso. También sería relevante que la plataforma de videoconferencia permita que el mediador pueda incorporar elementos de texto o audiovisuales, pues, como vimos, utilizar distintos tipos de lenguaje puede ser útil para gestionar desequilibrios de poder o sospechas de violencia dentro del proceso.

Por otra parte, como ya mencionamos, una de las labores que podrían verse dificultadas en la mediación en línea sería la firma de las actas de acuerdo, pero como también indicamos, existen distintas vías que podrían adoptarse para obtener la manifestación de voluntad de los participantes.

Tampoco existen antecedentes que confirmen la idea de que la mediación en línea no permita dar cumplimiento a los principios de la mediación de la Ley 19.968. Solo estimamos posible afirmar que eventualmente podría dificultarse el cumplimiento del principio de confidencialidad y de igualdad. En el primer caso, por la naturaleza propia de las comunicaciones digitales que las hace más vulnerables a intromisiones no deseadas. Sin embargo, como vimos al revisar las pautas internacionales, esto puede ser contrarrestado mediante planes de gestión de la seguridad, en los que, como mínimo, se debería incluir un análisis minucioso de las características de privacidad y seguridad de una tecnología. En cualquier caso, sería deber del mediador informar a los usuarios sobre las medidas que se han adoptado para asegurar su confidencialidad y seguridad y advertir los posibles riesgos asociados al uso de la tecnología. De este modo, el usuario contaría con los antecedentes necesarios para ejercer el principio de voluntariedad.

Luego, el principio de igualdad podría dificultarse si alguno de los participantes posee menos conocimientos digitales o un acceso inferior a conectividad digital. No obstante, en estos casos el mediador debería adoptar las medidas conducentes a obtener dicho equilibrio. Por ejemplo, sería conveniente que el mediador contara con recursos informativos para los usuarios acerca de la tecnología que se pretende utilizar y que pudiera ofrecerse la conectividad digital del respectivo centro. Sin embargo, si nada pudiera hacer el mediador para restablecer el equilibrio de poder entre los participantes, al igual que un caso presencial, tendría que frustrar el proceso. Por lo tanto, no se debe confundir una dificultad con la imposibilidad absoluta de dar 
cumplimiento a un determinado principio, porque en ningún caso la mediación en línea impide que los principios rectores de la mediación familiar puedan cumplirse en forma cabal. Tampoco es posible afirmar que la mediación presencial esté exenta de este tipo de dificultades.

\section{Conclusiones}

En este estudio demostramos que la mediación en línea puede favorecer el acceso de los usuarios a los servicios de mediación y evitar la discontinuidad del servicio cuando no es posible reunir físicamente a los participantes. Además, evidenciamos que la mediación en línea puede ofrecer otros beneficios a los usuarios, como favorecer su protagonismo y permitir que el servicio se adapte a sus necesidades o ritmo de vida. También se expuso que la mediación en línea puede beneficiar a los mediadores del Sistema de Mediación Familiar de Chile, al permitirles continuar trabajando cuando no es posible hacer mediaciones presenciales y resguardar su salud en el contexto de la pandemia de covid-19. Asimismo, mostramos que la mediación en línea no impide el desempeño adecuado de la labor mediadora o cumplir con los principios del proceso, sino que, por el contrario, existen circunstancias en que esta modalidad puede resultar más apropiada para dichos fines que un proceso presencial.

Por otra parte, al analizar el contexto social y tecnológico de Chile, resulta claro que la población posee altos niveles de acceso y uso de tecnologías digitales e internet, y que el Estado está haciendo inversiones importantes para que en el corto plazo la conectividad digital se extienda a todo el país.

Por todo lo señalado, sostenemos que no es razonable que solo se admita la concurrencia física al Sistema de Mediación Familiar de Chile, pues con este requisito excluyente, el servicio deja de responder de manera pertinente en los casos en que existen impedimentos de acceso físico a los centros de mediación. Este tipo de dificultades pueden originarse por diversos factores, como una emergencia sanitaria, distancias geográficas o factores económicos, entre otros. Además, cada vez será más común utilizar tecnologías digitales para comunicarse y la concurrencia física será menos significativa. Por lo tanto, es claro que la implementación de la mediación en línea en Chile es una solución adecuada para abordar las problemáticas familiares en el futuro cercano, de acuerdo con los desafíos que plantea la era digital y la diversidad familiar en Chile.

En atención a la experiencia internacional, también concluimos que se requiere establecer protocolos en los que se consignen estándares mínimos para asegurar una adecuada implementación de la mediación en línea. A partir de los antecedentes analizados, creemos que un protocolo para el Sistema Nacional de Mediación al menos debiese establecer: 
- Lineamientos para que el mediador pueda determinar la idoneidad de la mediación en línea. Para ello sería aconsejable considerar, como mínimo, las recomendaciones internacionales que se han dictado sobre este punto, las que recomiendan verificar que los usuarios tengan acceso a una tecnología adecuada, que todas las partes estén de acuerdo en seguir la mediación en línea, que todas posean capacidades suficientes para hacer uso de la tecnología, y que cualquier diferencia en las capacidades de las partes que usan la tecnología se pueda gestionar de manera efectiva.

- El uso preferencial de plataformas digitales de videoconferencia, ya que éstas permiten transmitir de manera integral los componentes de la comunicación.

- Que la plataforma de videoconferencia sea diseñada exclusivamente para el Sistema Nacional de Mediación Familiar, de manera que sea programada con altos estándares de seguridad para el tráfico de la información y la identificación de los participantes. Además, sería recomendable que se exija que la plataforma posea una interfaz de usuario de fácil manejo que solo permita acceder a las funcionalidades esenciales para la mediación. Por ejemplo, una casilla de verificación para manifestar la conformidad o rechazo de documentos, y que sea el mediador quien tenga el control exclusivo de las características complejas requeridas para el proceso, como la posibilidad de crear salas virtuales individuales o conjuntas, compartir o requerir documentos, controlar quién permanece en las salas, etcétera.

- Normas sobre la gestión de la seguridad, en las que como mínimo se consigne una revisión técnica anual de los estándares de seguridad que ofrece la plataforma de videoconferencia.

- Un plan para la gestión de fallas tecnologías, que como mínimo considere qué sucederá en los casos en que todos o alguno de los participantes tengan problemas de conectividad, o se presenten fallos en la plataforma de videoconferencia que impidan su uso o limiten sus funcionalidades.

- La manera en que se proveerá la conectividad digital a los usuarios de los centros de mediación licitados que cuenten con tecnologías digitales pero que no puedan costear los servicios de internet, y tampoco asistir a un centro de mediación para acceder a su conectividad o hacer un proceso presencial.

- La manera en que será manifestada la voluntad de aceptación o rechazo de los usuarios con respecto del consentimiento informado y las actas de acuerdo o frustración del proceso, y la forma en que serán exhibidos o remitidos al mediador los documentos necesarios para el desarrollo de la mediación. 
- El deber de informar a los usuarios sobre cómo la mediación se verá afectada por la distancia y el uso de tecnologías digitales, y cuáles son los compromisos que impone el uso de la tecnología en el proceso. Esta información debería ser entregada en la etapa de premediación a aquellos usuarios que consulten o soliciten acceder a esta modalidad. Para lo anterior, sería recomendable contar con infografías o videos que permitan complementar la información entregada a los usuarios por parte del mediador o administrativo del centro. Luego, esta información debería ser reiterada en el discurso inicial que ofrece el mediador antes de iniciar el proceso, y ser parte integrante del consentimiento informado que deben leer y firmar voluntariamente quienes deseen participar de él.

Con un protocolo que contemple al menos estas consideraciones, podemos asegurar que sí sería recomendable, e incluso necesaria, la implementación de la mediación en línea en el Sistema de Mediación Familiar de Chile. Por lo que venimos señalando, damos por confirmada la hipótesis de nuestra investigación y concluimos que no es correcto afirmar de manera categórica que la mediación en línea no sea recomendable para el Sistema de Mediación Familiar de Chile.

\section{Agradecimientos}

Agradezco a Jaqueline García Catalán y a Claudio Antonio Alarcón García, de Chile, y a Marta Inés González, de Argentina, por sus valiosos aportes a este trabajo.

\section{Referencias}

Alzate, Ramón (2008). «Mediación en Línea». Revista de Mediación, 1 (1): 6-15. Disponible en https://bit.ly/3d4xuGY.

Alzate, Ramón y Eduardo Vázquez (2013). Resolución de disputas en línea: Las claves de la mediación electrónica. Madrid: Reus.

EbNer, Noam (2012). «E-Mediation». En Mohamed Abdel, Ethan Katsh y Daniel Rainey (editores), Online dispute resolution: Theory and practice, a treatise on technology and dispute resolution. Portland: Eleven.

EBNer, Noam y John Zeleznikow (2015). «Fairness, trust and security in online dispute resolution». Journal of Public Law and Policy, 36 (2): 143-16o. Disponible en https://bit.ly/37xy7Yn.

LAVI, Dafna (2015). «Till death do us part: Online mediation as an answer to divorce cases involving violence». North Carolina Journal of Law \& Technology, 16 (2): 253310. Disponible en https://unc.live/37z3mm2.

Mediate BC (2012). Mediating from a distance: Suggested practice guidelines for family mediators. Vancouver. Disponible en https://bit.ly/2Y4I5gU. 
OrtegA, Rolando (2019). Mecanismos alternativos de resolución de conflictos por medios electrónicos. Barcelona: Bosch.

PeÑa, María (2014). El proceso de mediación, capacidad y habilidades del mediador. Madrid: Dykinson.

Poblet, Marta, Pablo Noriega, Josep Suquet, Silvia Gabarró y Josep Redorta (2011). «Tecnologías para la mediación en línea: Estado del arte, usos y propuestas». En Pompeu Casanovas, Jaume Magre y María Elena Lauroba (directores), Libro blanco de la mediación. Barcelona: Huygens.

Rodríguez, Jorge (2019). Migraciones internas en Chile, 1977-2017: Continuidad y cambio. Santiago: Cepal.

Wilson-Evered, Elisabeth, Deborah Macfarlane, John Zeleznikow y Mark Thomson (2011). "Towards an online family dispute resolution service in Australia». En Marta Poblet (editora), Mobile technologies for conflict management: Online dispute resolution, governance, participation. Barcelona: Springer. DOI: 10.1007/978-94-007-1384-0.

\section{Sobre la autora}

Sofía Andrea Alarcón García es abogada. Licenciada en Ciencias Jurídicas por la Universidad Católica de Temuco, Chile. Magíster en Resolución Colaborativa de Conflictos por la Universidad Católica de Temuco. Su correo electrónico es sag.abogada@gmail.com. (D) https://orcid.org/oooo-0003-2655-7512. 


\title{
REVISTA CHILENA DE DERECHO Y TECNOLOGÍA
}

La Revista de Chilena de Derecho y Tecnología es una publicación académica semestral del Centro de Estudios en Derecho Informático de la Facultad de Derecho de la Universidad de Chile, que tiene por objeto difundir en la comunidad jurídica los elementos necesarios para analizar y comprender los alcances y efectos que el desarrollo tecnológico y cultural han producido en la sociedad, especialmente su impacto en la ciencia jurídica.

\author{
EDITOR GENERAL \\ Daniel Álvarez Valenzuela \\ (dalvarez@derecho.uchile.cl) \\ SITIO WEB \\ rchdt.uchile.cl \\ CORREO ELECTRÓNICO \\ rchdt@derecho.uchile.cl \\ LICENCIA DE ESTE ARTÍCULO \\ Creative Commons Atribución Compartir Igual 4.o Internacional
}

\begin{abstract}
La edición de textos, el diseño editorial
y la conversión a formatos electrónicos de este artículo

estuvieron a cargo de Tipográfica

(www.tipografica.io).
\end{abstract}

